\title{
RNAfamProb Plus NeoFold: Estimations of Posterior Probabilities on RNA Structural Alignment and RNA Secondary Structures with Incorporating Homologous-RNA Sequences
}

\author{
Masaki Tagashira ${ }^{1,2, *}$ and Kiyoshi Asai ${ }^{1,2, *}$ \\ ${ }^{1}$ Department of Computational Biology and Medical Sciences, University of Tokyo, Chiba, 277-8561, Japan and \\ ${ }^{2}$ Artificial Intelligence Research Center, AIST, Tokyo, 135-0064, Japan. \\ *To whom correspondence should be addressed. \\ Associate Editor: $X X X X X X X$ \\ Received on XXXXX; revised on XXXXX; accepted on XXXXX
}

\begin{abstract}
Motivation: The simultaneous optimization of the sequence alignment and secondary structures among $R N A s$, structural alignment, has been required for the more appropriate comparison of functional $n c R N A s$ than sequence alignment. Pseudo-probabilities given RNA sequences on structural alignment have been desired for more-accurate secondary structures, sequence alignments, consensus secondary structures, and structural alignments. However, any algorithms have not been proposed for these pseudo-probabilities. Results: We invented the RNAfamProb algorithm, an algorithm for estimating these pseudo-probabilities. We performed the application of these pseudo-probabilities to two biological problems, the visualization with these pseudo-probabilities and maximum-expected-accuracy secondary-structure (estimation). The RNAfamProb program, an implementation of this algorithm, plus the NeoFold program, a maximumexpected-accuracy secondary-structure program with these pseudo-probabilities, demonstrated prediction accuracy better than three state-of-the-art programs of maximum-expected-accuracy secondary-structure while demanding running time far longer than these three programs as expected due to the intrinsic serious problem-complexity of structural alignment compared with independent secondary structure and sequence alignment. Both the RNAfamProb and NeoFold programs estimate matters more accurately with incorporating homologous-RNA sequences.

Availability: The source code of each of these two programs is available on each of "https://github.com/heartsh/rnafamprob" and "https://github.com/heartsh/neofold".

Contact: "tagashira masaki_17@stu-cbms.k.u-tokyo.ac.jp" and "asai@k.u-tokyo.ac.jp".
\end{abstract}

Supplementary information: Supplementary data are available at Bioinformatics online.

\section{Introduction}

A large quantity of RNAs not translated into proteins (= ncRNAs) being functional have been discovered through sequencing technology including high-throughput sequencing technology (Maxam and Gilbert, 1977; Bentley et al., 2008). These RNAs are related to various biological processes such as epigenetic silencing (Pasmant et al., 2011), splicing regulation (Ji et al., 2003), translational control (Long and Caceres, 2009), apoptosis regulation, and cell cycle control (Kino et al., 2010). However, a large number of these RNAs are functionally unknown. RNAs fold into 3D structures while taking less free energy (= FE). Sets of BasePairings (= BPs) in these structures, secondary structures (= SSs), should be considered together with RNA sequences when measuring the similarity of functional ncRNAs because both BP and unpaired bases play roles in biological processes (Wu et al., 1991).

The optimization of residue alignments among biomolecules, sequence alignment (Gotoh, 1982; Altschul et al., 1997; Katoh and Standley, 2013) (= SA), is required for the comparison of biomolecules mainly whose residues play roles in biological processes such as proteins and DNAs. The simultaneous optimization of the SA and SSs among RNAs, STructural Alignment (Sankoff, 1985) (= STA), has been required for the more appropriate comparison of functional ncRNAs. Pseudo-probabilities given RNA sequences on STA have been desired for more-accurate SSs, SAs, consensus SSs (= optimizations of aligning BP base-pairs among RNAs = CSSs) (Bernhart et al., 2008), and STAs. (Hamada et al., 2009c,a, 2011; Sato et al., 2012) However, any algorithms have not been proposed for estimating these pseudo-probabilities. Therefore, 


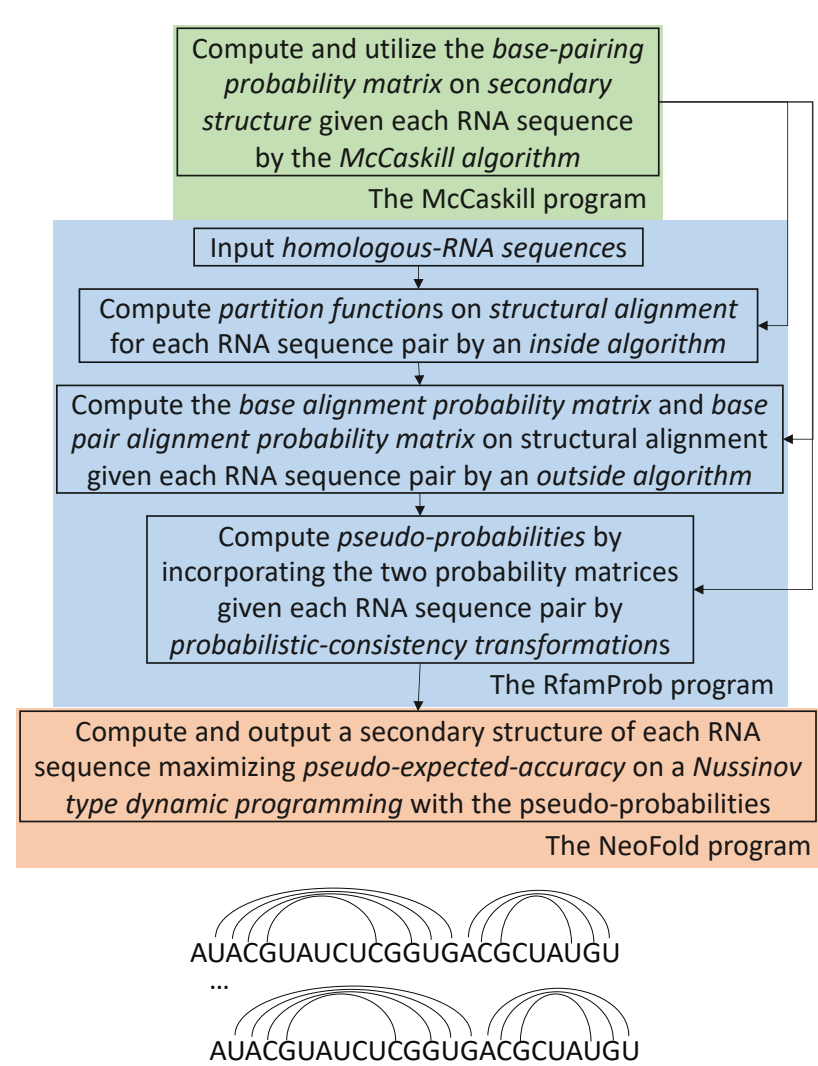

Fig. 1. The workflow of our proposed method of maximum-expected-accuracy secondarystructure estimation, the Neofold suite. This suite combines the RNAfamProb, McCaskill, and NeoFold programs. The RNAfamProb program estimates pseudo-base-pairingprobabilities more accurate than base-pairing probabilities estimated by the McCaskil program by considering the structural alignment between two homologous-RNA sequences. The McCaskill program helps the RNAfamProb program finish computations in realistic time by filtering in possible base-pairings on an RNA sequence. The Neofold program estimates secondary structures more accurately by using these pseudo-probabilities instea of these probabilities.

we invented the RNAfamProb algorithm, an algorithm for estimating pseudo-probabilities given RNA sequences on STA. We performed the application of these pseudo-probabilities to two biological problems, visualization with these pseudo-probabilities and Maximum-ExpectedAccuracy (= MEA) SS (Hamada et al., 2009b) (estimation). The RNAfamProb program, an implementation of this algorithm, plus the NeoFold program, an MEA SS program with these pseudo-probabilities, demonstrated prediction accuracy better than three state-of-the-art MEASS-programs while demanding running time far longer than these three programs as expected due to the intrinsic serious problem-complexity of STA compared with independent SS and SA. Both the RNAfamProb and NeoFold programs estimate matters more accurately with incorporating homologous-RNA sequences.

\section{Methods}

2.1 Our proposed method of maximum-expected-accuracy secondary structure

The workflow of our proposed MEA-SS-method, the NeoFold suite, is shown in Figure 1.

\subsection{Structural alignment}

An STA between two RNA sequences $\boldsymbol{R} \boldsymbol{N} \boldsymbol{A}, \boldsymbol{R} \boldsymbol{N} \boldsymbol{A}^{\prime}$ is defined as $\mathbb{S T} \mathbb{A}_{\mathbb{R N A}:=\left[R N A, R N A^{\prime}\right]}:=\left[S S_{R N A}, S S_{R N A^{\prime}}, S A_{\mathbb{R N A}}\right]$ where
(A)

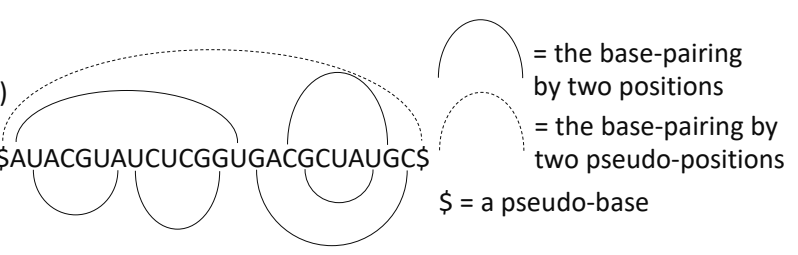

(B)

A secondary structure $\boldsymbol{S} \boldsymbol{S}_{\text {RNA }}$

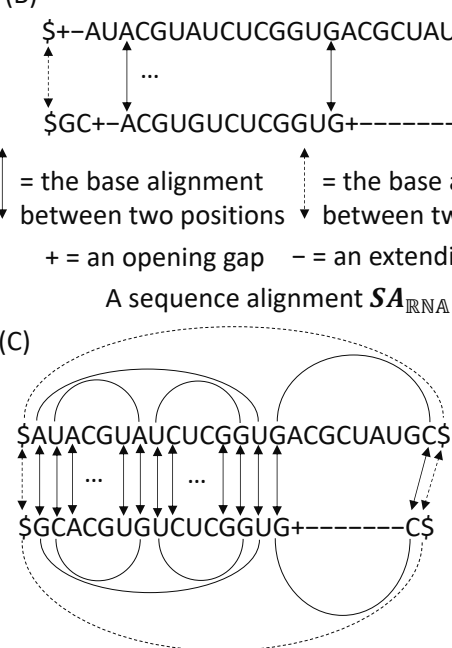

A structural alignment $\mathbb{S} \mathbb{T} \mathbb{A}_{\mathbb{R N A}}$

Fig. 2. An example of each of (A) a secondary structure $S S_{R N A}$, (B) sequence alignment $\boldsymbol{S} \boldsymbol{A}_{\mathbb{R N A}}$, and (C) structural alignment $\mathbb{S T A} A_{\mathbb{R N}}$

$\boldsymbol{S} \boldsymbol{S}_{\boldsymbol{R} \boldsymbol{N} \boldsymbol{A}}$ is defined as an SS of $\boldsymbol{R} \boldsymbol{N} \boldsymbol{A}$ and $\boldsymbol{S} \boldsymbol{A}_{\mathbb{R N A}}$ an SA of $\mathbb{R N A}$ The pseudo-positions $0, N+1$ are BP in any $\boldsymbol{S S}_{\boldsymbol{R} \boldsymbol{N} \boldsymbol{A}}$ where $N$ is defined as the length of $\boldsymbol{R} \boldsymbol{N} \boldsymbol{A}$. Each of the pseudo-position pairs $[0,0],[N+1, M+1]$ is aligned in any $\boldsymbol{S} \boldsymbol{A}_{\mathbb{R N A}}$ where $M$ is defined as the length of $\boldsymbol{R} \boldsymbol{N} \boldsymbol{A}^{\prime}$. Two position pairs $[i, j],[k, l] ; i, j, k, l \in \mathfrak{N}, 0 \leq$ $i<j \leq N+1,0 \leq k<l \leq M+1$ are corresponding if and only if each of $[i, j],[k, l]$ are $\mathrm{BP}$ and $[i, j],[k, l]$ are aligned. A position pair must be BP if this pair is aligned with a BP position pair. Only SS without any pseudoknots and collinear SAs are considered in this pape because considerations of these SSs and SAs lead to larger time and space complexities. (Sato et al., 2011; Bradley et al., 2009)

A pairwise CFG (= pair-CFG) describing STAs $\mathbb{C F} \mathbb{G}^{\text {sta }}$ is defined as

$\mathbb{C F} \mathbb{G}^{\text {sta }}=\left[\mathcal{V}^{\text {sta }}, \mathcal{A}^{\text {sta }}, \mathcal{R}^{\text {sta }}, v^{\mathrm{s}, \mathrm{sta}}\right] ; \mathcal{V}^{\text {sta }}:=\left\{v^{\mathrm{s}, \mathrm{sta}}, v^{\sigma}\right\}$

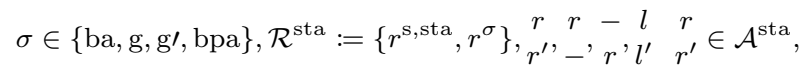

$r^{\mathrm{s}, \mathrm{sta}}:=v^{\mathrm{s}, \mathrm{sta}} \rightarrow v^{\sigma}, r^{\mathrm{ba}}:=v^{\mathrm{ba}} \rightarrow v^{\mathrm{s}, \mathrm{sta}}{ }_{{ }^{\prime}}{ }^{\prime}\left|\epsilon, r^{\mathrm{g}}:=v^{\mathrm{g}} \rightarrow v^{\sigma}{ }_{-}^{r}\right| \epsilon$,

$r^{\mathrm{g} \prime}:=v^{\mathrm{g} \prime} \rightarrow v_{r}^{\sigma}{ }_{r}^{-}\left|\epsilon, r^{\mathrm{bpa}}:=v^{\mathrm{bpa}} \rightarrow v^{\mathrm{s}, \mathrm{sta}}{ }_{l^{\prime}}{ }^{\mathrm{s}, \mathrm{sta}}{ }_{r^{\prime}}{ }^{\prime}\right| \epsilon$

where each of $l, r, l^{\prime}, r^{\prime}$ is defined as a base and - a gap. $\mathcal{V}^{\text {sta }}$ is a set of variables, $\mathcal{R}^{\text {sta }}$ a set of transitions each from a variable to variables (= rules), and $v^{\mathrm{s}, \mathrm{sta}}$ a start variable. This pair-CFG is equivalent to the pair-CFG described in Dowell and Eddy (2006). An example of each of $\boldsymbol{S S}_{\boldsymbol{R N A}}, \boldsymbol{S} \boldsymbol{A}_{\mathbb{R N A}}, \mathbb{S T} \mathbb{A}_{\mathbb{R N A}}$ is shown on each of (A), (B), and (C) in Figure 2.

\subsection{Boltzmann distribution and posterior probabilities}

$\mathfrak{S T A}_{\mathbb{R N A}}$ is defined as the STA space of $\mathbb{R N A}$. The Boltzmann distribution of $\mathbb{S T A} \in \mathfrak{S T} \mathfrak{A}_{\mathbb{R N A}}$ is defined as $p_{\mathbb{S T A} \mathbb{A}}:=\frac{\exp \left(-\beta^{\text {sta }} f e_{\mathbb{S T A}}\right)}{Z^{\text {sta }}} ; Z^{\text {sta }}:=$ 
$\sum_{\mathbb{S T A}} \exp \left(-f e_{\mathbb{S T A}}\right), \beta^{\text {sta }} \in \mathfrak{R}^{+}$where $f e_{\mathbb{S T A}}$ is defined as the FE of STA and $\mathfrak{R}^{+}$the positive-real-number space excluding $0 . Z^{\text {sta }}$ is the partition function $(=\mathrm{PF})$ on $\mathfrak{S} \mathfrak{T A}_{\mathbb{R N A}}$ and $\beta^{\text {sta }}$ a scale parameter of $f e_{\mathbb{S T A}}$.

Definition 2.1. Each of the base alignment probability $(=B A P)$ matrix $(=B A P M)$ on STA and base pair alignment probability $(=B P A P)$ matrix (= BPAPM) given $\mathbb{R N A}$ is defined as each of $\boldsymbol{P}_{\mid \mathbb{R N A}}^{\mathrm{ba}, \text { sta }}:=$ $\left(p_{0 \leq u \leq N+1,0 \leq v \leq M+1 \mid \mathbb{R N A}}^{\mathrm{ba}, \mathrm{sta}}:=\sum_{\mathbb{S T A} \mid I_{\mathbb{S T A} u v}^{\mathrm{ba}}=1} p_{\mathbb{S T A} \mathbb{A}}\right), \boldsymbol{P}_{\mid \mathbb{R N A}}^{\mathrm{bpa}}:=$ $\left(p_{i j k l \mid \mathbb{R N A}}^{\mathrm{bpa}}:=\sum_{\mathbb{S T A} \mid I_{\mathbb{S T A} A j k l}^{\mathrm{bpa}}=1} p_{\mathbb{S T A} \mathbb{A}}\right)$ where $I_{\mathbb{S T A} \text { uv }}^{\mathrm{ba}}$ is defined as 1 if the two positions $u, v$ are aligned and unpaired in $\mathbb{S T A}$ and 0 otherwise and $I_{\mathbb{S T A} A j k l}^{\mathrm{bpa}} 1$ if the two position pairs $[i, j],[k, l]$ are corresponding in $\mathbb{S T A}$ and 0 otherwise.

2.4 Formulation of structural-alignment free energy $f e_{\mathbb{S T A}}$

$f e_{\mathbb{S T A}}$ can be formulated as

$f e_{\mathbb{S T} \mathbb{A}}=f e_{\boldsymbol{S} S}+f e_{\boldsymbol{S} \boldsymbol{S}^{\prime}}+s\left(f e^{\mathrm{ba}, \mathrm{sta}}+f e^{\mathrm{g}}+f e^{\mathrm{bpa}}\right) ; s \in \mathfrak{R}^{+}$,

$f e^{\mathrm{ba}, \mathrm{sta}}:=-\sum_{u v \mid I_{\mathbb{S T A} \mathbb{A}}^{\mathrm{ba}}=1} \ln \frac{p_{u v}^{\mathrm{ba}, \mathrm{sta}}}{p_{u v}^{\mathrm{ba}, \mathrm{sta}, \mathrm{r}}}, f e^{\mathrm{g}}:=-n_{\mathbb{S T \mathbb { A }}}^{\mathrm{og}} p^{\mathrm{og}}-n_{\mathbb{S T A}}^{\mathrm{eg}} p^{\mathrm{eg}}$,

$f e^{\mathrm{bpa}}:=-\sum_{i j k l \mid I_{\mathbb{S T A} i j k l}^{\mathrm{bpa}}=1} \ln \frac{p_{i j k l}^{\mathrm{bpa}}}{p_{i j k l}^{\mathrm{bpa}, \mathrm{r}}}$

where $f e_{\boldsymbol{S} S}$ is defined as the FE of $\boldsymbol{S S}$, each of $p_{u v}^{\mathrm{ba}, \mathrm{sta}}, p_{i j k l}^{\mathrm{ba}, \mathrm{sta}, \mathrm{r}}$ the prior BAP of the two positions $u, v$ on STA on each of the two assumptions in whether $\mathbb{R N A}$ is correlated or uncorrelated, each of $p_{i j k l}^{\mathrm{bpa}}, p_{i j k l}^{\mathrm{bpa}, \mathrm{r}}$ the prior BPAP of the two position pairs $[i, j],[k, l]$ corresponding on each of these two assumptions, each of $n_{\mathbb{S T A}}^{\mathrm{og}}, n_{\mathbb{S T A}}^{\text {eg }}$ the number of each of opening and extending gaps in STA and each of $p^{\mathrm{og}}, p^{\mathrm{eg}}$ a penalty of each of two opening and extending gaps. $s$ is the scale parameter of $f e^{\mathrm{ba} \text {,sta }}+f e^{\mathrm{g}}+$ $f e^{\text {bpa }}$ relative to $f e_{\boldsymbol{S} S}+f e_{\boldsymbol{S} \boldsymbol{S}^{\prime}}$.

$p_{u v}^{\mathrm{ba}, \text { sta }}$ can be formulated as $p_{u v}^{\mathrm{ba}, \text { sta }} \approx \frac{f_{r n a u}^{\mathrm{ba}, \mathrm{sta} n a_{v}^{\prime}}}{\sum_{r r^{\prime}} f_{r r^{\prime}}^{\mathrm{ba}, \mathrm{sta}}}$ where $f_{r r^{\prime}}^{\mathrm{ba} \text { sta }}$ is defined as the frequency of $r, r^{\prime}$ aligned in STAs for estimating $p_{u v}^{\mathrm{ba}, \text { sta }}$, $r n a_{u}$ the $u$-th base of $\boldsymbol{R N A}$, and $r n a_{v}^{\prime}$ the $v$-th base of $\boldsymbol{R} \boldsymbol{N} \boldsymbol{A}^{\prime} . p_{i j k l}^{\text {bpa' }}$ can be formulated similarly to $p_{u v}^{\mathrm{ba}, \mathrm{sta}} \cdot p_{u v}^{\mathrm{ba}, \mathrm{sta}, \mathrm{r}}$ can be formulated as $p_{u v}^{\mathrm{ba}, \mathrm{sta}, \mathrm{r}} \approx \frac{f_{r n a_{u}} f_{r n a_{v}^{\prime}}}{\sum_{r r^{\prime}} f_{r} f_{r^{\prime}}}$ where $f_{r}$ is defined as the frequency of $r$ in STAs for estimating $p_{u v}^{\text {ba,sta, } \mathrm{r}}$

Probabilities $p_{u v}^{\mathrm{ba}, \mathrm{sta}}, p_{i j k l}^{\mathrm{bpa}}, p_{u v}^{\mathrm{ba}, \mathrm{sta}, \mathrm{r}}, p_{i j k l}^{\mathrm{bpa}, \mathrm{r}}$ estimated are available as the RIBOSUM STA FE matrices. (Klein and Eddy, 2003) On the other hand, $f e_{S S}$ is available as the parameters of the Turner model, a nearest-neighbor approximation model for RNA SS FE on thermodynamics. (Turner and Mathews, 2010) $p_{i j \mid \boldsymbol{R} N \boldsymbol{A}}^{\mathrm{bp}, \mathrm{ss}}$ have been utilized as components of $f e_{S S}$ on computing on STA due to computations simplified more though the suitability of $p_{i j \mid R N A}^{\mathrm{bp}, \mathrm{ss}}$ to $f e_{S S}$ has not been discussed. (Hofacker et al., 2004; Do et al., 2008) In this study, the Turner model is combined with the two RIBOSUM STA FE 80-65 matrices for preventing the accuracy of $\boldsymbol{P}_{\mid \mathbb{R N A}}^{\mathrm{ba}, \mathrm{sta}}, \boldsymbol{P}_{\mid \mathbb{R N A}}^{\mathrm{bpa}}$ from losing.

2.5 Inside-outside algorithm for computing base alignment probability matrix and base pair alignment probability matrix $\boldsymbol{P}_{\mid \mathbb{R N A}}^{\text {ba,sta }}, \boldsymbol{P}_{\mid \mathbb{R N A}}^{\text {bpa }}$

Each of $p_{u v \mid \mathbb{R N A}}^{\mathrm{ba}, \mathrm{sta}}, p_{i j k l \mid \mathbb{R N A}}^{\mathrm{bpa}}$ can be formulated as each of

$$
\begin{aligned}
p_{u v \mid \mathbb{R N A}}^{\mathrm{ba}, \mathrm{sta}} & =\frac{\sum_{\mathbb{S T A} \mid I_{\mathbb{S T A} \mathbb{A}}^{\mathrm{ba}}=1} \exp \left(-\beta^{\mathrm{sta}} f e_{\mathbb{S T A}}\right)}{Z^{\mathrm{sta}}}, \\
p_{i j k l \mid \mathbb{R N A}}^{\mathrm{bpa}} & =\frac{\sum_{\mathbb{S T A} \mid I_{\mathbb{S T A} \mathbb{A} k l l}^{\mathrm{bpa}}=1} \exp \left(-\beta^{\mathrm{sta}} f e_{\mathbb{S T A}}\right)}{Z^{\mathrm{sta}}} .
\end{aligned}
$$

Thus, first $Z^{\text {sta }}$ and then $p_{u v \mid \mathbb{R N A}}^{\mathrm{ba}, \mathrm{sta}}, p_{i j k l \mid \mathbb{R N A}}^{\mathrm{bpa}}$ are formulated recursively, which leads to Algorithm 1 .
$\boldsymbol{P}_{\mid \mathbb{R N A}}^{\text {ba,sta }}, \boldsymbol{P}_{\mid \mathbb{R N A}}^{\mathrm{bpa}}$ can be computed by Algorithm 1 with the time and space complexities $O\left(N^{4} M^{4}\right), O\left(N^{2} M^{2}\right)$. This algorithm is the simultaneous solution of the Durbin (forward-backward) algorithm (Durbin et al., 1998) and McCaskill (inside-outside $=\mathrm{IO}$ ) algorithm (McCaskill, 1990) and the IO algorithm version of the Sankoff algorithm, a maximum-likelihood (= ML) STA algorithm (Sankoff, 1985), as expected. An analysis of these two complexities is described in

Algorithm 1 An inside-outside algorithm for computing a base alignment probability matrix and base pair alignment probability matrix $\boldsymbol{P}_{\mid \mathbb{R N A}}^{\mathrm{ba}, \mathrm{sta}}, \boldsymbol{P}_{\mid \mathbb{R N A}}^{\mathrm{bpa}}$

1: function ioAlgo4BapmAndBpapm(RNA)

2: Compute partition functions on an inside algorithm according to Supplementary section $1 / /$ Inside step.

3: // Outside step. Partition functions other than partition function $Z_{i j k l}^{\text {sta,bpa }}$ need to be recomputed for exploiting more sparsity of partition functions.

4: Compute base alignment probabilities and base pair alignment probabilities $p_{u v \mid \mathbb{R N A}}^{\mathrm{ba}, \mathrm{sta}}, p_{i j k l \mid \mathbb{R N A}}^{\mathrm{bpa}}$ on an outside algorithm while recomputing partition functions other than $Z_{i j k l}^{\text {sta,bpa }}$ on this inside algorithm according to Supplementary section 2 and Supplementary section 1

5: $\quad$ return $\left[\boldsymbol{P}_{\mid \mathbb{R N A}}^{\text {ba,sta }}, \boldsymbol{P}_{\mid \mathbb{R N A}}^{\text {bpa }}\right]$

Supplementary section 2.

2.6 Inside-outside algorithm for computing base alignment probability matrix and base pair alignment probability matrix $\boldsymbol{P}_{\mid \mathbb{R N A}}^{\text {ba,sta }}, \boldsymbol{P}_{\mid \mathbb{R N A}}^{\text {bpa }}$ approximate

Algorithm 1 is not practical because of the time and space complexitie of this algorithm $O\left(N^{4} M^{4}\right), O\left(N^{2} M^{2}\right)$. IO algorithms for computing $\boldsymbol{P}_{\mid \mathbb{R N A}}^{\mathrm{ba}, \mathrm{sta}}, \boldsymbol{P}_{\mid \mathbb{R N A}}^{\mathrm{bpa}}$ approximate (or sparse) with less time and space complexities can be gained by adding restrictions to (or sparsifying) $S \mathcal{S} \mathcal{A}_{\mathbb{R N A}}$.

Two restrictions frequently added to this space are the minimum BPP restriction (Sato et al., 2012) and maximum-gap-number restriction (Torarinsson et al., 2007). $p_{i j \mid \mathrm{BN} \boldsymbol{A}}^{\mathrm{bp}, \mathrm{ss}}$ is defined as the BPP of the two positions $i, j$ given $\boldsymbol{R} \boldsymbol{N} \boldsymbol{A}$ on SS, computed by the McCaskill algorithm. The minimum-BPP restriction allows any two positions $i, j$ to base-pair only when $p_{i j, \mathrm{bs}}^{\mathrm{bp}, \mathrm{ss}}$ is greater than or equal to a minimum BPP $p^{\mathrm{bp}, \mathrm{ss}, \mathrm{m}} \in \mathfrak{R}^{+} ; 0 \leq p^{\mathrm{bp}, \mathrm{ss}, \mathrm{m}} \leq 1$. The maximum-gap-number restriction allows any STA only when the gap number in STA is less than or equal to a maximum gap-number $g^{\mathrm{m}} \in \mathfrak{N} ;|N-M| \leq g^{\mathrm{m}}$.

The probable-STA restriction is defined as the combination of the minimum-BPP and maximum-gap-number restrictions. $\mathcal{S} \mathcal{T} \mathcal{A}_{\mathbb{R N A}}^{\mathrm{r}}$ is defined as the STA space gained by adding the probable-STA restriction to any $\mathbb{S T A}$. Each of $\boldsymbol{P}_{\mid \mathbb{R N A}}^{\mathrm{ba}, \text { sta, a }}, \boldsymbol{P}_{\mid \mathbb{R N A}}^{\mathrm{bpa} \text { a a }}$ is defined as each of the approximate BAPM and BPAPM gained by replacing $\mathcal{S T} \mathcal{A}_{\mathbb{R N A}}$ with $\mathcal{S} \mathcal{T} \mathcal{A}_{\mathbb{R N A}}^{\mathrm{r}}$ in Definition 2.1. Algorithm 1 with this restriction is virtually with the time and space complexities $O\left(L^{2}\right), O(L) ; L:=\max (N, M)$ if $p^{\mathrm{bp}, \mathrm{ss}, \mathrm{m}}$ takes a sufficiently large value and $g^{\mathrm{m}}$ takes a sufficiently small value.

\subsection{Probabilistic-consistency transformation}

A Probabilistic-Consistency Transformation (= PCT) is the transformation of a probability between a biomolucule or biomolecules and each homologous biomolecule into a pseudo-probability. (Do et al., 2005) This pseudo-probability contains information about all utilized homologous biomolecules. $\boldsymbol{R F}$ is defined as an RNA family, a sequence of homologous-RNA sequences each with a length of at most $N$. Each

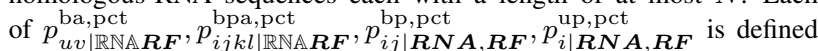
of $p_{u v \mid \mathbb{R N A R F}}, p_{i j k l \mid \mathbb{R N A R F}}, p_{i j \mid \boldsymbol{R N A} \boldsymbol{R} \boldsymbol{R},}, p_{i \mid \boldsymbol{R} N \boldsymbol{R} \boldsymbol{R} \boldsymbol{R} \text { is defined }}$
as a pseudo-BAP, pseudo-BPAP, pseudo-BPP, and pseudo-unpairingprobability gained by performing a PCT between $\mathbb{R N A}$ or $R N A$ an 
$\boldsymbol{R F} . p_{u v}^{\text {ba,pct }}$ can be formulated as

$p_{u v \mid \mathbb{R N A R} \boldsymbol{R}}^{\mathrm{ba}, \mathrm{pct}}=\frac{p_{u v \mid \mathbb{R N A}}^{\mathrm{ba}, \mathrm{sta}, \mathrm{a}}+\sum_{\boldsymbol{R N} \boldsymbol{A}^{\prime \prime} f}\left(p_{u f \mid \mathbb{R N A} \mathbb{A}^{\prime}}^{\mathrm{ba}, \mathrm{sta}, \mathrm{a}} p_{f v \mid \mathbb{R N A} \mathbb{A}^{\prime \prime}}^{\mathrm{ba}, \mathrm{sta}, \mathrm{a}}\right)}{R-1} ;$

$R:=t(\boldsymbol{R F}), \mathbb{R N A}^{\prime}:=\left[\boldsymbol{R N} \boldsymbol{A}, \boldsymbol{R} \boldsymbol{N} \boldsymbol{A}^{\prime \prime}\right], \mathbb{R N A}^{\prime \prime}:=\left[\boldsymbol{R} \boldsymbol{N} \boldsymbol{A}^{\prime \prime}, \boldsymbol{R N} \boldsymbol{A}^{\prime}\right.$

where $\boldsymbol{R} \boldsymbol{N} \boldsymbol{A}^{\prime \prime} \in \boldsymbol{R} \boldsymbol{F} \backslash\left(\boldsymbol{R N} \boldsymbol{A}, \boldsymbol{R} \boldsymbol{N} \boldsymbol{A}^{\prime}\right), 0 \leq f \leq t\left(\boldsymbol{R} \boldsymbol{N} \boldsymbol{A}^{\prime \prime}\right)-1$ and $t(\boldsymbol{R F})$ is defined as a function returning the type (or size) of $\boldsymbol{R} \boldsymbol{F}$. $p_{i j p a, p c t}^{\text {bet }}$ can be formulated similarly to $p^{\mathrm{ba}, \mathrm{pct}}$, $p^{\mathrm{bp}, \mathrm{pct}}$ can be formulated as

$$
\begin{aligned}
& p_{i j \mid \boldsymbol{R} \boldsymbol{N} \boldsymbol{A}, \boldsymbol{R} \boldsymbol{F}}^{\mathrm{bp}, \mathrm{pct}}= \begin{cases}p_{i j \mid \boldsymbol{R} \boldsymbol{N} \boldsymbol{A}, \boldsymbol{R} \boldsymbol{F}} & \left(p_{i j \mid \boldsymbol{R} \boldsymbol{N} \boldsymbol{A}, \boldsymbol{R} \boldsymbol{F}}^{\mathrm{bpct}^{\prime}}>0\right) \\
p_{i j \mid \boldsymbol{R} \boldsymbol{R} \boldsymbol{A} \boldsymbol{A}}^{\mathrm{bp}^{\prime}} & (\text { otherwise })\end{cases} \\
& p_{i j \mid \boldsymbol{R} \boldsymbol{N} \boldsymbol{A}, \boldsymbol{R} \boldsymbol{F}}^{\mathrm{bp}_{\mathrm{pct}}{ }^{\prime}}=\frac{\sum_{\boldsymbol{R} \boldsymbol{N} \boldsymbol{A}^{\prime} \in \boldsymbol{R} \boldsymbol{F} \backslash(\boldsymbol{R N} \boldsymbol{A})} p_{i j \mid \mathbb{R N A}}^{\mathrm{bp}, \mathrm{sta}, \mathrm{a}}}{R-1}, \\
& p_{i j \mid \mathbb{R N A}}^{\mathrm{bp}, \mathrm{sta}, \mathrm{a}}:=\sum_{k l} p_{i j k l \mid \mathbb{R N A}}^{\mathrm{bpa}, \mathrm{a}} .
\end{aligned}
$$

$p_{i \mid \boldsymbol{R} N \boldsymbol{A}, \boldsymbol{R} \boldsymbol{F}}^{\text {up,pet }}$ can be formulated as

$$
\begin{aligned}
& p_{i \mid \boldsymbol{R N} \boldsymbol{A}, \boldsymbol{R} \boldsymbol{F}}^{\mathrm{up}, \mathrm{pct}}:=\frac{\sum_{\boldsymbol{R} \boldsymbol{N} \boldsymbol{A}^{\prime} \in \boldsymbol{R} \boldsymbol{F} \backslash(\boldsymbol{R} \boldsymbol{N} \boldsymbol{A})} p_{i \mid \mathbb{R N A}}^{\mathrm{up}, \mathrm{sta}, \mathrm{a}}}{R-1} ; \\
& p_{u \mid \mathbb{R N A}}^{\mathrm{up}, \mathrm{sta}, \mathrm{a}}:=1-\sum_{0 \leq u^{\prime}<u}\left(p_{u^{\prime} u \mid \mathbb{R N A}}^{\mathrm{bp}, \mathrm{sta}, \mathrm{a}}\right)-\sum_{u<u^{\prime \prime} \leq N+1} p_{u u^{\prime \prime} \mid \mathbb{R N A} \mathbb{A}}^{\mathrm{bp}, \mathrm{sta}, \mathrm{a}} .
\end{aligned}
$$

$\boldsymbol{R N} \boldsymbol{A}_{\boldsymbol{R} \boldsymbol{F}}$ is defined as a sequence of pairs of the RNA sequences in $\boldsymbol{R} \boldsymbol{F}$ Pseudo-probability matrices

$$
\begin{aligned}
& \boldsymbol{P}_{\mid \mathbb{R N A} \in \boldsymbol{R} N \boldsymbol{A}_{\boldsymbol{R} \boldsymbol{F}}, \boldsymbol{R F}}^{\mathrm{ba}, \mathrm{pct}}:=\left(p_{u v \mid \mathbb{R N A} \boldsymbol{R} \boldsymbol{F}}^{\mathrm{ba} p \mathrm{pct}}\right), \\
& \boldsymbol{P}_{\mid \mathbb{R N A} \in \boldsymbol{R} N \boldsymbol{A}_{\boldsymbol{R} \boldsymbol{F}}, \boldsymbol{R} \boldsymbol{F}}^{\mathrm{bpa}, \mathrm{pct}}:=\left(p_{i j k l \mid \mathbb{R N A} \boldsymbol{R} \boldsymbol{F}}^{\text {bpa,pct }}\right) \text {, } \\
& \boldsymbol{P}_{\mid \boldsymbol{R N} \boldsymbol{A} \in \boldsymbol{R} \boldsymbol{F}, \boldsymbol{R} \boldsymbol{F}}^{\mathrm{bp}, \mathrm{pct}}:=\left(p_{i j \mid \boldsymbol{R N} \boldsymbol{A}, \boldsymbol{R} \boldsymbol{F}}^{\mathrm{bp}, \mathrm{pct}}\right), \\
& \boldsymbol{P}_{\mid \boldsymbol{R} \boldsymbol{N} \boldsymbol{A} \in \boldsymbol{R} \boldsymbol{F}, \boldsymbol{R} \boldsymbol{F}}^{\text {up,pct }}:=\left(p_{i \mid \boldsymbol{R} \boldsymbol{N} \boldsymbol{A}, \boldsymbol{R} \boldsymbol{F}}^{\text {up,pct }}\right)
\end{aligned}
$$

can be computed by the RNAfamProb algorithm, Algorithm 2.

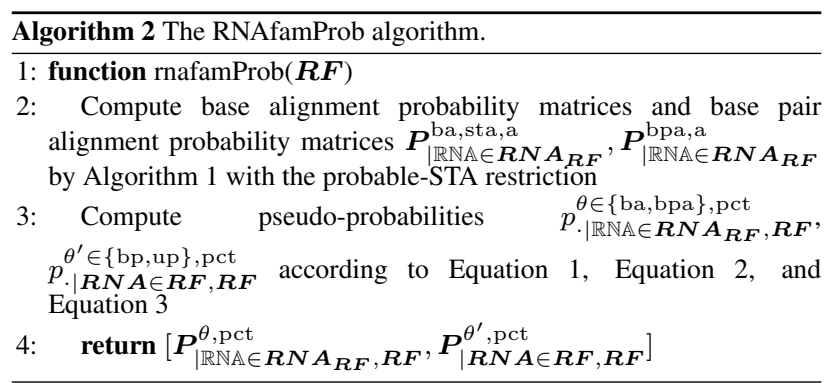

2.8 Maximum-expected-accuracy secondary structure incorporating single homologous-RNA sequence

The accuracy of $\boldsymbol{S S}$ against $\mathbb{S T A}$ is defined as

$$
\begin{aligned}
& a_{\boldsymbol{S S S T A}}:=\alpha_{1} T P^{\mathrm{ss}}+\alpha_{2} T N^{\mathrm{ss}}-\alpha_{3} F P^{\mathrm{ss}}-\alpha_{4} F N^{\mathrm{ss}} ; \\
& \alpha_{h:=1, \cdots, 4} \in \mathfrak{R}^{+}, T P^{\mathrm{ss}}:=\sum_{i j}\left(I_{\boldsymbol{S} \boldsymbol{S} i j}^{\mathrm{bp}} I_{\mathbb{S T A} A j}^{\mathrm{bp}}\right), \\
& T N^{\mathrm{ss}}:=\sum_{i}\left[\left(1-I_{\boldsymbol{S} \boldsymbol{S} i}^{\mathrm{bp}}\right)\left(1-I_{\mathbb{S T A} A}^{\mathrm{bp}}\right)\right], \\
& F P^{\mathrm{ss}}:=\sum_{i}\left[I_{\boldsymbol{S} \boldsymbol{S} i}^{\mathrm{bp}}\left(1-I_{\mathbb{S T A} A}^{\mathrm{bp}}\right)\right], F N^{\mathrm{ss}}:=\sum_{i}\left[\left(1-I_{\boldsymbol{S} \boldsymbol{S} i}^{\mathrm{bp}}\right) I_{\mathbb{S T A} A}^{\mathrm{bp}}\right]
\end{aligned}
$$

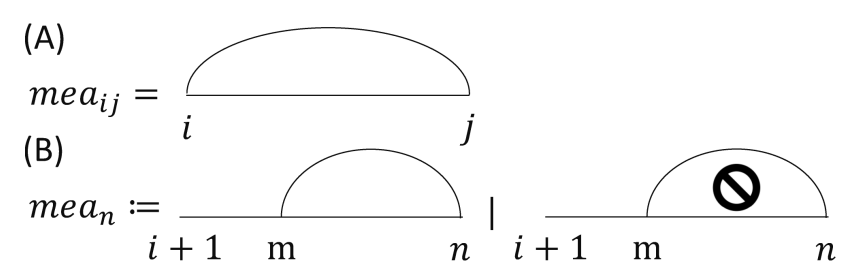

Fig. 3. An interpretation of each of (A) the formulation of maximum expected accuracy $m e a_{i j}$ and (B) definition of maximum expected accuracy $m e a_{n}$.

where $I_{\mathbb{S T A} i j}^{\mathrm{bp}}$ is defined as 1 if the two positions $i, j$ are BP in $\mathbb{S T A}$ and 0 otherwise, $I_{\boldsymbol{S} S i}^{\mathrm{bp}} 1$ if $i$ is BP in $\boldsymbol{S S}$ and 0 otherwise, and $I_{\mathbb{S T A} i}^{\mathrm{bp}} 1$ if $i$ is BP in $\mathbb{S T A}$ and 0 otherwise. Each of $T P^{\mathrm{ss}}, T N^{\mathrm{ss}}, F P^{\mathrm{ss}}, F N^{\mathrm{ss}}$ is the number of each of true positives (=TPs), true negatives $(=\mathrm{TNs})$, false positives $(=$ FPs), and false negatives (= FNs). Each of $\alpha_{h}$ a scale parameter for each of $T P^{\mathrm{ss}}, T N^{\mathrm{ss}},-F P^{\mathrm{ss}},-F N^{\mathrm{ss}}$.

$a_{S S S T A}$ and accuracy $a_{S S S T A}^{\gamma \mathrm{c}}:=\gamma T P^{\mathrm{ss}}+T N^{\mathrm{ss}} ; \gamma:=\frac{\alpha_{1}+2 \alpha_{4}}{\alpha_{2}}$ are equivalent in terms of SS accuracy. Expected accuracy $\left(=\stackrel{\alpha_{2}+\alpha_{3}}{=}\right.$ $\mathbb{E}_{\mathbb{S T A}}\left[a_{S S \mathbb{S T A}}^{\gamma \mathrm{c}}\right]$ can be formulated as

$$
\begin{aligned}
& \mathbb{E}_{\mathbb{S T A} \mathbb{A}}\left[a_{\boldsymbol{S S S T A}}^{\gamma \mathrm{c}}\right]=\gamma \sum_{i j \mid I_{S}^{\mathrm{bp} i j}=1}\left(p_{i j \mid \mathbb{R N A}}^{\mathrm{bp}, \mathrm{sta}}\right)+\sum_{i \mid I_{S \boldsymbol{S} i}^{\mathrm{bp}}=0} p_{i \mid \mathbb{R N A}}^{\mathrm{up}, \mathrm{sta}} ; \\
& p_{i j \mid \mathbb{R N A}}^{\mathrm{bp}, \mathrm{sta}}:=\sum_{k l} p_{i j k l \mid \mathbb{R N A}}^{\mathrm{bpa}} \\
& p_{u \mid \mathbb{R N A}}^{\mathrm{up}, \mathrm{sta}}:=1-\sum_{u^{\prime}}\left(p_{u^{\prime} u \mid \mathbb{R N A}}^{\mathrm{bp}, \mathrm{sta}}\right)-\sum_{u^{\prime \prime}} p_{u u^{\prime \prime} \mid \mathbb{R N A}}^{\mathrm{bp}, \mathrm{sta}} .
\end{aligned}
$$

A proof of each of these relationship and formulation is described in each of Supplementary section 4 and Supplementary section 5.

Definition 2.2. The MEA SS of $\boldsymbol{R N A}$ is defined as $\widehat{\boldsymbol{S S}}_{\boldsymbol{R N A} \boldsymbol{A}}:=$ $\underset{S S}{\arg \max } \mathbb{E}_{\mathbb{S T A} \mathbb{A}}\left[a_{S S S T \mathbb{A}}^{\gamma \mathrm{c}}\right]$

mea $_{i j}$ is defined as the EA of $\widehat{\boldsymbol{S S}}_{\boldsymbol{R N A} \boldsymbol{A}_{i j}}$ in the case in which the two positions $i, j$ are BP where $\boldsymbol{R} \boldsymbol{N} \boldsymbol{A}_{i j}$ is defined as the substring of $\boldsymbol{R} \boldsymbol{N} \boldsymbol{A}$ between $i, j$ inclusive of $i, j \cdot \max _{S S} \mathbb{E}_{\mathbb{S T A}}\left[a_{S S S T A}^{\gamma \mathrm{c}}\right]$ is gained from the fact $\max _{\boldsymbol{S} S} \mathbb{E}_{\mathbb{S T A} \mathbb{A}}\left[a_{S \boldsymbol{S S T A}}^{\gamma \mathrm{c}}\right], m e a_{0, N+1}$ are equal for any $\mathbb{R N A}$. $m e a_{i j}$ can be formulated as

$$
\begin{aligned}
& \text { mea }_{i j}=m e a_{j-1}+\gamma p_{i j \mid \mathbb{R N A}}^{\mathrm{bp}, \mathrm{sta}} ; \\
& \text { mea }_{i<n<j}:=\max \left\{\begin{array}{l}
\max _{i<m<n}\left(m_{i<a_{m-1}}+\text { mea }_{m n}\right) \\
m e a_{n-1}+p_{n \mid \mathbb{R N A}}^{\text {up,sta }}
\end{array}\right.
\end{aligned}
$$

An interpretation of each of the formulation of $m e a_{i j}$ and definition of $m e a_{n}$ is shown on each of (A) and (B) in Figure 3. It should be noted Equation 4 is a Nussinov type dynamic programming (= DP) recursive equation (Nussinov et al., 1978). mea $_{i}$ is formulated (or initialized) as mea $_{i}=0 . \widehat{\boldsymbol{S S}}_{\boldsymbol{R} \boldsymbol{N} \boldsymbol{A}}$ can be computed with the time and space complexities $O\left(N^{4}\right), O\left(N^{2}\right)$ by Supplementary algorithm 2. A proof of these two complexities is described in Supplementary section 6.

\subsection{Pseudo-maximum-expected-accuracy secondary structure incorporating multiple homologous-RNA sequences}

$\widehat{S S}_{\boldsymbol{R N A}}^{\text {pct }}$ is defined as the MEA SS gained by replacing $p_{i j \mid \mathbb{R N A}}^{\mathrm{bp} \text {,sta }}$ with $p_{i j \mid \boldsymbol{R} \boldsymbol{N} \boldsymbol{A}, \boldsymbol{R} \boldsymbol{F}}^{\text {bp,pct }}$ and $p_{i \mid \mathbb{R N A}}^{\text {up,sta }}$ with $p_{i \mid \boldsymbol{R} \boldsymbol{N} \boldsymbol{A}, \boldsymbol{R} \boldsymbol{F}}^{\text {up,pct }}$ in Definition 2.2 $\widehat{S S}_{\boldsymbol{R N A}}^{\text {pt }}$ can be computed by the NeoFold algorithm, Supplementary algorithm 3. This algorithm is virtually with the time and space complexities $O\left(N^{2}\right), O(N)$ if the number of positions able to base-pair with a position is sufficiently small. 
2.10 Relationship between NeoFold algorithm and other maximum-expected-accuracy secondary-structure algorithms

The NeoFold algorithm can be considered as the CONTRAfold algorithm, an MEA SS algorithm (Do et al., 2006), improved by replacing probabilities on SS with pseudo-probabilities on STA and modifying slightly accuracy. This algorithm maximizes EA $2 \gamma^{\mathrm{c}} \sum_{i j \mid I_{S S i j}^{\mathrm{bp}}=1}\left(p_{i j \mid \boldsymbol{R} \boldsymbol{N} \boldsymbol{A}}^{\mathrm{bp}, \mathrm{ss}}\right)+\sum_{i \mid I_{S \boldsymbol{S} i}^{\mathrm{bp}}=0} p_{i \mid \boldsymbol{R} \boldsymbol{N} \boldsymbol{A}}^{\mathrm{up}, \mathrm{ss}} ; \gamma^{\mathrm{c}} \in \mathfrak{R}^{+}$ Thus, this algorithm counts twice TPs and FNs compared with the NeoFold algorithm. On the other hand, the NeoFold algorithm can be considered as the CentroidFold algorithm, an MEA SS algorithm (Hamada et al., 2009b), improved by replacing probabilities on SS with pseudo-probabilities on STA and modifying drastically accuracy. This algorithm maximizes EA

$\mathbb{E}_{\boldsymbol{S} \boldsymbol{S}^{\prime} \in \mathfrak{S S}_{\boldsymbol{R} N A}}\left[\alpha_{1}^{\mathrm{c}} T P^{\mathrm{ss}, \mathrm{c}}+\alpha_{2}^{\mathrm{c}} T N^{\mathrm{ss}, \mathrm{c}}-\alpha_{3}^{\mathrm{c}} F P^{\mathrm{ss}, \mathrm{c}}-\alpha_{4}^{\mathrm{c}} F N^{\mathrm{ss}, \mathrm{c}}\right] ;$ $\alpha_{h}^{\mathrm{c}} \in \mathfrak{R}^{+}, T P^{\mathrm{ss}, \mathrm{c}}:=\sum_{i j}\left(I_{\boldsymbol{S} \boldsymbol{S} i j}^{\mathrm{bp}} I_{\boldsymbol{S} \boldsymbol{S}^{\prime} i j}^{\mathrm{bp}}\right)$,

$T N^{\mathrm{ss}, \mathrm{c}}:=\sum_{i j}\left[\left(1-I_{\boldsymbol{S} \boldsymbol{S} i j}^{\mathrm{bp}}\right)\left(1-I_{\boldsymbol{S}^{\prime} i j}^{\mathrm{bp}}\right)\right]$,

$F P^{\mathrm{ss}, \mathrm{c}}:=\sum_{i j}\left[I_{\boldsymbol{S} \boldsymbol{S} i j}^{\mathrm{bp}}\left(1-I_{\boldsymbol{S} \boldsymbol{S}^{\prime} i j}^{\mathrm{bp}}\right)\right], F N^{\mathrm{ss}, \mathrm{c}}:=\sum_{i j}\left[\left(1-I_{\boldsymbol{S} \boldsymbol{S} i j}^{\mathrm{bp}}\right) I_{\boldsymbol{S}^{\prime}{ }^{\prime} i j}^{\mathrm{bp}}\right]$

where $\mathfrak{S S}_{R N A}$ is defined as the SS space of $\boldsymbol{R N A}$. This algorithm overcounts TNs compared with the NeoFold algorithm, especially when RNA sequences are long, since at most one position $j$ can base-pair with a position $i$. The NeoFold algorithm counts TPs, TNs, FPs, and FNs equally, i.e. the contribution of these four numbers to the accuracy of this algorithm is unbiased.

The CentroidHomFold algorithm, an MEA SS algorithm incorporating homologous-RNA sequences via PCT similarly to the NeoFold algorithm, considers STAs by decomposing $p_{i j k l \mid \mathbb{R N A}}^{\mathrm{bpa}}$ as $p_{i j k l \mid \mathbb{R N A}}^{\mathrm{bpa}} \approx$ $p_{i j \mid R \boldsymbol{N} \boldsymbol{A}}^{\mathrm{bp}, \mathrm{ss}}+p_{i k \mid \mathbb{R N A}}^{\mathrm{ba}, \mathrm{sa}} p_{j l \mid \mathrm{RN}}^{\mathrm{ba}, \mathrm{sa}} p^{\mathrm{bp}, \mathrm{ss}}$

where $p$ a,sand is defined as the algorithm (Durbin et al., 1998). Mathematical decompositions like this decomposition are frequently utilized in many RNA informatics algorithms including the CentroidAlign algorithm $(=$ an MEA SA algorithm considering STA) (Hamada et al., 2009a), CentroidAliFold algorithm (= an MEA CSS algorithm) (Hamada et al., 2011), and DAFS algorithm (= an MEA STA algorithm) (Sato et al., 2012) because precise considerations of STAs demand $\boldsymbol{P}_{\mid \mathbb{R N A}}^{\mathrm{ba}, \text { sta }}, \boldsymbol{P}_{\mid \mathbb{R N A}}^{\mathrm{bpa}}$, whose computations have been proposed in this paper. When the probable-STA restriction is removed completely on the RNAfamProb algorithm, the CentroidHomFold algorithm is an approximate version of the NeoFold algorithm.

The NeoFold algorithm incorporates precisely homologous-RNA sequences via STA whereas SS algorithms so far do not incorporate these sequences or avoid STA via mathematical-decomposition techniques as shown in Figure 4.

\section{Results and discussions}

3.1 Algorithm implementations and environments for running programs

Each of the RNAfamProb, NeoFold, and McCaskill algorithms was implemented on the Rust programming language as the version 0.1 .0 of each of the RNAfamProb, NeoFold, and McCaskill programs. These three programs employ multi-threading as much as possible for achieving running time as fast as possible. The source code of each of these three programs is available on each of "https://github.com/heartsh/rnafamprob", "https://github.com/heartsh/neofold", and "https://github.com/heartsh/rnaalgos". Programs were run on a computer composed of the "Intel Xeon CPU E5-2680 v2" CPU with 20 CPU logical cores and the clock rate $2.80[\mathrm{GHz}]$ and $64[\mathrm{~GB}]$ of RAM unless environments for running programs are specified.

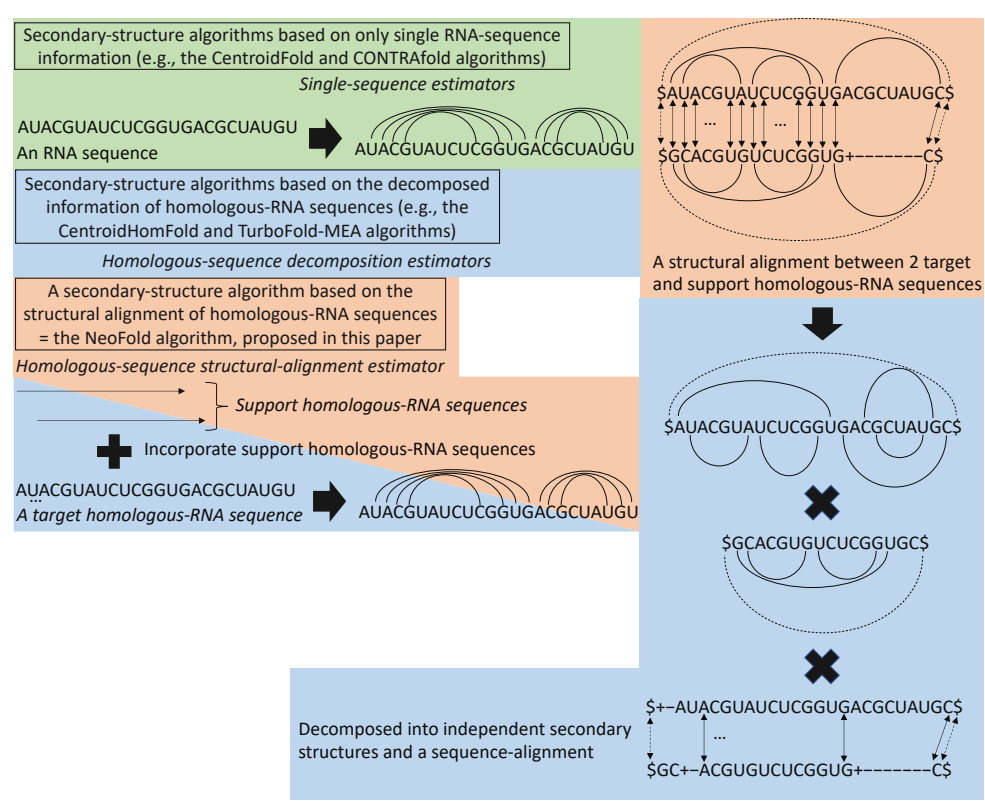

Fig. 4. The comparison among three types of secondary-structure estimator. Singlesequence and homologous-sequence decomposition estimators are proposed in many studies so far. Homologous-sequence structural-alignment estimator has been proposed for the first time in this paper. The ascending order of the expected reliability of these three types is single sequence estimator $\rightarrow$ homologous-sequence decomposition estimator $\rightarrow$ homologous-sequence structural-alignment estimator.

\subsection{Visualization with pseudo-probabilities $p_{i j k l \mid \mathbb{R N A R F}}^{\text {bpa,pct }}, p_{i j \mid \boldsymbol{R} \boldsymbol{N} \boldsymbol{A}, \boldsymbol{R} \boldsymbol{F}}^{\mathrm{bp}, \mathrm{pct}}$}

3.2.1 Visualization of pseudo-base-pair-alignment-probability $p_{i j k l \mid \text { bpt }}^{\text {bpa, }}$ as reliability of two corresponding position-pairs $[i, j],[k, l]$ $p_{i j k l \mid \mathbb{R N A R F}}^{\mathrm{bpa} \text { pct }}$ can be used as the reliability of the two corresponding position-pairs $[i, j],[k, l]$ as in Figure 5. The ascending order of the expected reliability of the CSS on each of (A), (B), and (C) in Figure 5 is $(\mathrm{A}) \rightarrow(\mathrm{B}) \rightarrow(\mathrm{C})$. This order is consistent with the observed reliability of these three CSSs.

3.2.2 Visualization of pseudo-base-pairing-probability $p_{i j \mid R N}^{\text {bp,pct }}$ as reliability of two BP positions $i, j$ Six examples of each of $\boldsymbol{P}_{\mid \boldsymbol{R} \boldsymbol{N} \boldsymbol{A}}^{\mathrm{bp}, \mathrm{ss}}, \boldsymbol{P}_{\mid \boldsymbol{R} \boldsymbol{N} \boldsymbol{A}, \boldsymbol{R} \boldsymbol{F}}^{\mathrm{bp}}$ are shown in Figure 6 Two positions $i, j$ having $p_{i j \mid \boldsymbol{R N} \boldsymbol{A}, \boldsymbol{R} \boldsymbol{F}}^{\mathrm{bp}, \mathrm{pct}}<p_{i j \mid \boldsymbol{R} \boldsymbol{N} \boldsymbol{A}}^{\mathrm{bp}, \mathrm{ss}}$ mean the BP by $i, j$ is supported less by the consideration of homologous-RNA sequences. On the other hand, two positions $i, j$ having $p_{i j \mid \boldsymbol{R N} \boldsymbol{A}, \boldsymbol{R F}}^{\mathrm{bp}, \mathrm{pct}}>p_{i j \mid \boldsymbol{R N} \boldsymbol{A}}^{\mathrm{bp}, \mathrm{ss}}$ mean the BP by $i, j$ is supported more by this consideration. $p_{i j \mid R N}^{\text {bp } p \text { pct }}$ can be used as the reliability of the two BP positions $i, j$ as well as $p_{i j \mid \boldsymbol{R} \boldsymbol{N} \boldsymbol{A}}^{\mathrm{bp}, \mathrm{ss}}$ as in Supplementary figure 4.

\subsection{Comparison of NeoFold algorithm with four maximum-expected-accuracy secondary-structure algorithms}

The RNA sequences each having the Rfam database as a source of the ncRNA family to which this sequence belongs and a reference SS of this sequence were collected from the RNA STRAND database, a database of SSs (Andronescu et al., 2008), as test set 1 . These sequences in this set have no bases except for adenines, guanines, cytosines, and uracils. The SSs in this set have no pseudoknots. Ten RNA sequences and the SS of each of these ten sequences were sampled from a ncRNA family when this family has 11 or more RNA-sequences. The number of the RNA sequences and range of the RNA sequence lengths on each ncRNA family in this set are shown in Supplementary table 2. 


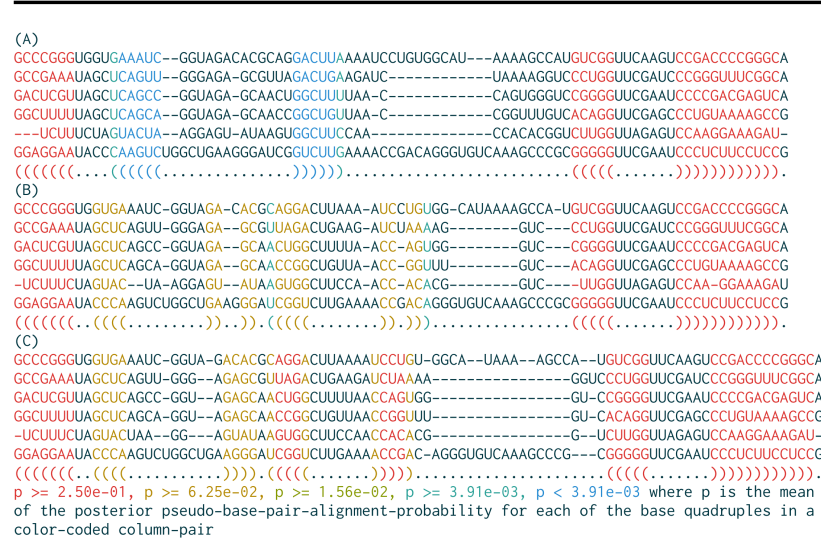

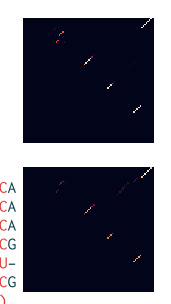

1
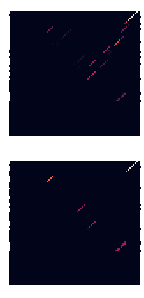

2

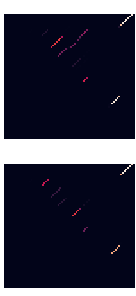

3

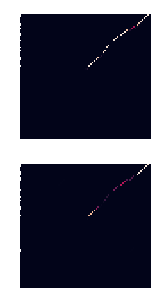

4

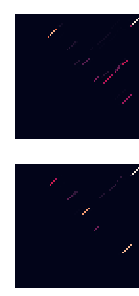

5

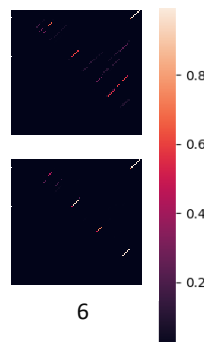

Fig. 5. Three examples of a consensus secondary structure color-coded based on pseudo- Fig. 6. Six examples of each of (upper) a base-pairing probability matrix and (lower)

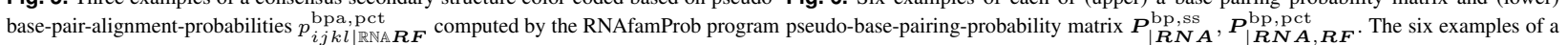
plus the McCaskill program (= the RNAfamProb suite) each with the sequence alignment base-pairing probability matrix $\boldsymbol{P}_{\mid \mathbb{R N A} \mathrm{ss}}^{\mathrm{bp}}$ were computed by the McCaskill program. The among six RNA sequences sampled from the tRNA family in the Rfam database. G-INS-i program, an maximum-likelihood sequence alignment program (Katoh and by the RNAfamProb suite with the seven parameter values same as Figure 5. Each of these Standley, 2013), used for computing this structure by the RNAalifold program, an 12 matrices has the indices 1,1 at the upper left corner of this matrix. These six sequences maximum-likelihood consensus-secondary-structure program (Bernhart et al, 2008). (B) are the same as Figure 5.

The consensus secondary structure with this alignment computed by the MAFFT X-INSi program, an maximum-likelihood sequence alignment program considering structural alignment, used for computing this structure by the RNAalifold program. (C) The reference consensus secondary structure derived from the Rfam database with the reference sequence alignment among these six sequences derived from this database. The RNAfamProb program was run with the seven parameter values in Supplementary table 1. A "()" means at least two position pairs in the column pair corresponding to these two parentheses are corresponding.
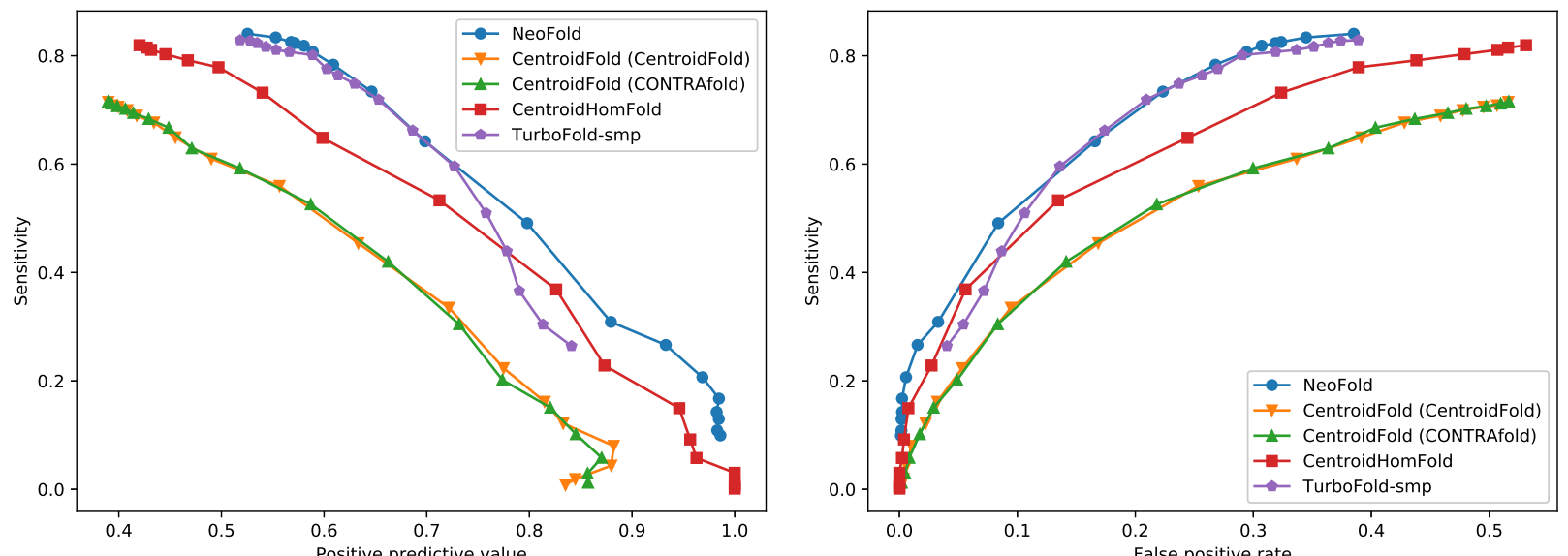

Fig. 7. The plot of positive predictive values versus sensitivities by varing the value of a Fig. 8 . The plot of false positive rates versus sensitivities by varing the value of a parameter parameter determining the balance between positive and negative among $W$ for each of (A) determining the balance between positive and negative among $W$ for each of (A) the the NeoFold suite and (B) CentroidFold, (C) CentroidHomFold, and (D) TurboFold-smp NeoFold suite and (B) CentroidFold, (C) CentroidHomFold, and (D) TurboFold-smp programs on test set 1. (A) The RNAfamProb program was run with the seven parameter programs on test set 1 . These suite and three programs were run with the parameter values values same as Figure 5. (B, C) The CentroidFold and CentroidHomFold programs were same as Figure 7.

run with the default parameter values of these two programs. The name in a "()" is of the

algorithm run by the CentroidFold program. (D) The TurboFold-smp program was run with

the number of times of the TurboFold iteration $\eta \leftarrow 1$ and default values of the parameters other than $\eta$.

The version 0.0 .15 of the CentroidFold program provides the CentroidFold and CONTRAfold algorithms. The version 0.0.15 of the CentroidHomFold program provides the CentroidHomFold algorithm. The version 6.0.1 of the TurboFold-smp program provides the TurboFold-MEA algorithm, an MEA SS algorithm with incorporating homologous-RNA sequences via the iterative refinement of BPPs on SS with the help of ML pairwise SAs, with multi-threading. (Tan et al., 2017) The principle of each of the CentroidHomFold and TurboFold-MEA algorithms is shown in Supplementary section 7. A distinct difference between these two algorithms and the NeoFold algorithm is whether posterior BPPs for computing MEA SSs are on STA or not.

Each of a positive predictive value (= PPV), sensitivity, and false positive rate $(=\mathrm{FPR})$ is defined as each of

$$
P P V:=\frac{T P^{\mathrm{t}}}{T P^{\mathrm{t}}+F P^{\mathrm{t}}}, S:=\frac{T P^{\mathrm{t}}}{T P^{\mathrm{t}}+F N^{\mathrm{t}}}, F P R:=\frac{F P^{\mathrm{t}}}{T N^{\mathrm{t}}+F P^{\mathrm{t}}}
$$

where each of $T P^{\mathrm{t}}, T N^{\mathrm{t}}, F P^{\mathrm{t}}, F N^{\mathrm{t}}$ is defined as the number of each of TPs, TNs, FPs, and FNs on a test with test set 1. A TP on this test is 
Table 1. The running time and approximate time-complexity on an RNA family $\boldsymbol{R F}$ of each of the NeoFold suite and CentroidFold, CentroidHomFold, and TurboFold-smp programs on test set 1 . These suite and three programs were run for the value of a parameter determining the balance between positive and negative $2^{0}=1$. These suite and three programs were run on the "Intel Xeon CPU" CPU with 20 CPU logical cores and the clock rate $2.30[\mathrm{GHz}]$ and 32 [GB] of RAM with the parameter values same as Figure 7. The NeoFold suite and TurboFold-smp program were run with employing multi-threading as much as possible for achieving running time as fast as possible. The CentroidFold and CentroidHomFold programs were run with employing multi-processing as much as possible for achieving running time as fast as possible.

\begin{tabular}{l|l|l} 
Program/Suite & Running time $[\mathrm{s}]$ & Time complexity \\
\hline \hline NeoFold & 31652.036268 & $O\left(R N^{2}(R+N)\right)$ \\
CentroidFold (CentroidFold) & 8.569625 & $O\left(R N^{3}\right)$ \\
CentroidFold (CONTRAfold) & 8.698287 & $O\left(R N^{3}\right)$ \\
CentroidHomFold & 99.557175 & $O\left(R^{2} N^{3}\right)$ \\
TurboFold-smp & 485.652050 & $O\left(R N^{2}(R+N)\right)$
\end{tabular}

two positions BP on both two predicted and reference SSs. A TN on this test is a position unpaired on both two predicted and reference SSs. An FP on this test is a position BP on a predicted SS but unpaired on a reference SS. An FN on this test is a position unpaired on a predicted SS but BP on a reference SS. These four numbers are configured in the same way as $a_{S S \mathbb{S T A}}$

The plot of PPVs versus sensitivities by varing the value of a parameter determining the balance between positive and negative (e.g., $\gamma$ on the NeoFold program) among $W:=\left\{2^{w} \mid w \in\{-7, \ldots, 10\}\right\}$ for each of the RNAfamProb program plus the McCaskill and NeoFold programs (= the NeoFold suite), and CentroidFold, CentroidHomFold, and TurboFoldsmp programs on test set 1 is shown in Figure 7. The plot of FPRs versus sensitivities by varing this value among $W$ for each of these suite and three programs on test set 1 is shown in Figure 8. The more a PPV-versussensitivity curve faces the upper right corner of the plot space of this curve, the better the prediction accuracy of the estimator corresponding to this curve is. Similarly, the more a FPR-versus-sensitivity (or receiver operating characteristic $=$ ROC) curve faces the upper left corner of the plot space of this curve, the better the prediction accuracy of the estimato corresponding to this curve is. The running time and approximate timecomplexity of each of these suite and three programs on test set 1 is shown in Table 1.

The ascending order of the number of PFs computed by each of these suite and three programs is the CentroidFold program $\rightarrow$ the CentroidHomFold and TurboFold-smp programs $\rightarrow$ the NeoFold suite. Therefore, the ascending order of the complexities of the probabilities computed by each of these suite and three programs is the same as this order of the numbers of these PFs. Thus, it is expected the NeoFold suite is the best of these suite and three programs in terms of prediction accuracy but worst of these suite and three programs in terms of running time. The prediction accuracy ascending order of these suite and three programs is observed to be the CentroidFold program $\rightarrow$ the CentroidHomFold prgoram $\rightarrow$ the TurboFold-smp program $\rightarrow$ the NeoFold suite, as expected. On the other hand, the running time ascending order of these suite and three programs is observed to be the CentroidFold program $\rightarrow$ the CentroidHomFold program $\rightarrow$ the TurboFold-smp program $\rightarrow$ the NeoFold suite, as expected.

\section{Conclusions}

The RNAfamProb algorithm, an algorithm for estimating pseudoprobabilities given RNA sequences on STA, and NeoFold algorithm, an MEA SS algorithm with these pseudo-probabilities, have been invented. The RNAfamProb and NeoFold programs, an implementation of each of the RNAfamProb and NeoFold algorithms, demonstrated prediction accuracy better than three state-of-the-art MEA-SS-programs while demanding running time far longer than these three programs as

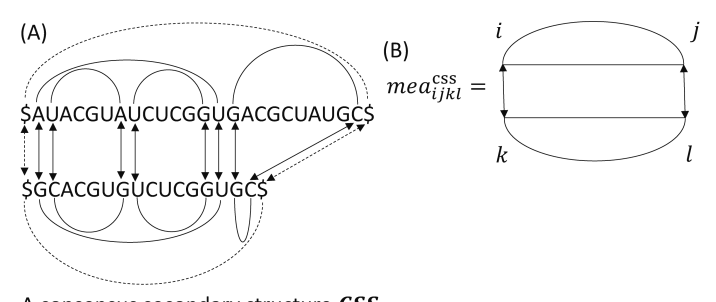

A consensus secondary structure $\boldsymbol{C} \boldsymbol{S} \boldsymbol{S}_{\mathbb{R N A}}$

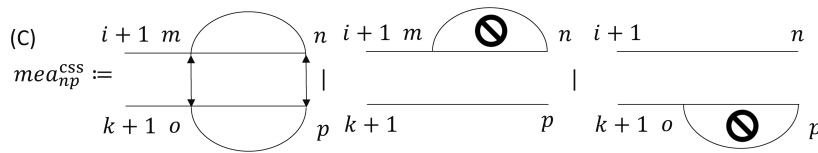

Fig. 9. (A) An example of a consensus secondary structure $\boldsymbol{C S} \boldsymbol{S}_{\mathbb{R N A}}$ and interpretation of each of (A) the formulation of maximum expected accuracy $m e a_{i j k l}^{\mathrm{css}}$ and (B) definition of maximum expected accuracy $m e a_{n p}^{\text {css }}$

expected due to the intrinsic serious problem-complexity of STA compared with independent SS and SA.

The RNAfamProb algorithm can be immediately applied to the CentroidAlign algorithm, CentroidAliFold algorithm, and DAFS algorithm for improving the prediction accuracy of these three algorithms.

Even a novel type of MEA CSS algorithm can be invented with the RNAfamProb algorithm. A CSS between a sequence pair $\mathbb{R N A}$ is defined as $\boldsymbol{C S} \boldsymbol{S}_{\mathbb{R N A}}:=\left(c s s_{i j k l}\right)$ where $c s s_{i j k l}$ is defined as 1 if the two position pairs $[i, j],[k, l]$ are corresponding and 0 otherwise. An example of a CSS $\boldsymbol{C S} \boldsymbol{S}_{\mathbb{R N A}}$ is shown on (A) in Figure 9. The MEA CSS between $\mathbb{R N A}$, $\widehat{\boldsymbol{C S S}}_{\mathbb{R N A}}$, can be computed according to

$m e a_{i j k l}^{\mathrm{css}}=m e a_{j-1, l-1}^{\mathrm{css}}+\gamma^{\mathrm{css}} p_{i j k l \mid \mathbb{R N A}}^{\mathrm{bpa}} ; \gamma^{\mathrm{css}} \in \mathfrak{R}^{+}$

$m e a_{n, k<p<l}^{\mathrm{css}}:=\max \left\{\begin{array}{l}\max _{m, k<o<p}\left(\operatorname{mea}_{m-1, o-1}^{\mathrm{css}}+m e a_{m n o p}^{\mathrm{css}}\right) \\ m e a_{n-1, p}^{\mathrm{css}}+p_{n \mid \mathbb{R N A}}^{\mathrm{up}, \mathrm{sta}} \\ m e a_{n, p-1}^{\mathrm{css}}+p_{p \mid \mathbb{R N A}}^{\mathrm{up}, \mathrm{Rs} \prime}\end{array}\right.$

$p_{v \mid \mathbb{R N A}}^{\mathrm{up}, \mathrm{sta}^{\prime}}:=1-\sum_{0 \leq v^{\prime}<v}\left(p_{v^{\prime} v \mid \mathbb{R N A}}^{\mathrm{bp}, \mathrm{sta} /}\right)-\sum_{v<v^{\prime \prime} \leq M+1} p_{v v^{\prime \prime} \mid \mathbb{R N A}}^{\mathrm{bp}, \mathrm{sta}^{\prime}}$,

$p_{k l \mid \mathbb{R N A}}^{\mathrm{bp}, \mathrm{sta} a}:=\sum_{i j} p_{i j k l \mid \mathbb{R N A}}^{\mathrm{bpa}}$

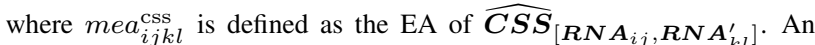
interpretation of each of the formulation of $m e a_{i j k l}^{\mathrm{css}}$ and definition of $m e a_{n p}^{\text {css }}$ is shown on each of (B) and (C) in Figure 9. $\widehat{\boldsymbol{C S S}}_{\mathbb{R N A}}$ with Equation 5 can be gained without any $\boldsymbol{S} \boldsymbol{A}_{\mathbb{R N A}}$, required by CSS programs so far such as the RNAalifold and CentroidAliFold programs. The quality of CSSs computed from these CSS programs has heavily depended on the quality of SAs supplied with these CSS programs for identifying candidates of two corresponding position-pairs $[i, j],[k, l]$. Therefore, the quality of MEA CSSs with Equation 5 will be generally superior to the quality of CSSs computed with these CSS programs.

The RNAfamProb algorithm plus the NeoFold algorithm will be able to estimate more-accurate SSs by incorporating SS probing data such as data from the icSHAPE (= one of experiments probing unpaired bases) (Spitale et al. 2015) of homologous-RNA sequences like the SuperFold algorithm, an SS algorithm with incorporating data from the SHAPE-MaP (= one of experiments probing unpaired bases) (Siegfried et al., 2014).

\section{Acknowledgements}

We thank the members of the Asai and Frith laboratories for discussing this study with us. Computations were partially performed on the NIG supercomputer at the ROIS National Institute of Genetics. 


\section{References}

Altschul, S. F. et al. (1997). Gapped BLAST and PSI-BLAST: a new generation of protein database search programs. Nucleic acids research, 25(17), 3389-402. Andronescu, M. et al. (2008). RNA STRAND: The RNA Secondary Structure and Statistical Analysis Database. BMC Bioinformatics, 9(1), 340.

Bentley, D. R. et al. (2008). Accurate whole human genome sequencing using reversible terminator chemistry. Nature, $\mathbf{4 5 6}(7218), 53-59$.

Bernhart, S. H. et al. (2008). RNAalifold: improved consensus structure prediction for RNA alignments. BMC Bioinformatics, $\mathbf{9}(1), 474$.

Bradley, R. K. et al. (2009). Fast statistical alignment. PLoS Computational Biology,

5(5).
Do, C. B. et al. (2005). ProbCons: Probabilistic consistency-based multiple sequence alignment. Genome research, 15(2), 330-40.

Do, C. B. et al. (2008). A max-margin model for efficient simultaneous alignment Do, C. B. et al. (2008). A max-margin model for efficient simultan
and folding of RNA sequences. Bioinformatics, 24(13), i68-i76.

Do, C. B., Woods, D. A., and Batzoglou, S. (2006). CONTRAfold: RNA secondary Do, C. B., Woods, D. A., and Batzoglou, S. (2006). CONTRAfold: RNA secondary
structure prediction without physics-based models. Bioinformatics, 22(14), e90structure

Dowell, R. D. and Eddy, S. R. (2006). Efficient pairwise RNA structure prediction and alignment using sequence alignment constraints. BMC bioinformatics, 7, 400. Durbin, R. et al. (1998). Biological sequence analysis.

Gotoh, O. (1982). An improved algorithm for matching biological sequences. Journal of Molecular Biology, 162(3), 705-708.

Hamada, M. et al. (2009a). CentroidAlign: fast and accurate aligner for structured RNAs by maximizing expected sum-of-pairs score. Bioinformatics (Oxford, England), 25(24), 3236-43.

Hamada, M. et al. (2009b). Prediction of RNA secondary structure using generalized centroid estimators. Bioinformatics, 25(4), 465-473.

Hamada, M. et al. (2009c). Predictions of RNA secondary structure by combining Hamada, M. et al. (2009c). Predictions of RNA secondary structure by co
homologous sequence information. Bioinformatics, 25(12), i330-i338.

homologous sequence information. Bioinformatics, 25(12), i330-i338.
Hamada, M. et al. (2011). Improving the accuracy of predicting secondary structure Hamada, M. et al. (2011). Improving the accuracy of predicting seconda

for aligned RNA sequences. Nucleic Acids Research, 39(2), 393-402.
Hofacker, I. L. et al. (2004). Alignment of RNA base pairing probability matrices. Hofacker, I. L. et al. (2004). Alignmento

Bioinformatics, 20(14), 2222-2227.
$\mathrm{Ji}$, P. et al. (2003). MALAT-1, a novel noncoding RNA and thymosin $\beta 4$ predict metastasis and survival in early-stage non-small cell lung cancer. Oncogene, 22(39), 8031-8041.

Katoh, K. and Standley, D. M. (2013). MAFFT Multiple Sequence Alignment Software Version 7: Improvements in Performance and Usability. Molecular
Biology and Evolution, 30(4), 772-780.

Kino, T. et al. (2010). Noncoding RNA gas5 is a growth arrest- and starvationassociated repressor of the glucocorticoid receptor. Science signaling, 3(107) ra8.

Klein, R. J. and Eddy, S. R. (2003). RSEARCH: Finding homologs of single structured RNA sequences. BMC Bioinformatics, 4(1), 44

Long, J. C. and Caceres, J. F. (2009). The SR protein family of splicing factors: master regulators of gene expression. The Biochemical journal, 417(1), 15-27.

Maxam, A. M. and Gilbert, W. (1977). A new method for sequencing DNA (DNA chenistry/dimethyl sulfate cleavage/hydrazine/piperidine). Biochemistry, 74(2), chenistry/. 564 .

McCaskill, J. S. (1990). The equilibrium partition function and base pair binding probabilities for RNA secondary structure. Biopolymers, 29(6-7), 1105-1119. probabilities for RNA secondary structure. Biopolymers, 29(6-7), 1105-1119.
Nussinov, R., Pieczenik, G., Griggs, J. R., and Kleitman, D. J. (1978). Algorithm Nussinov, R., Pieczenik, G., Griggs, J. R., and Kleitman, D. J. (1978). Algorith
for Loop Matchings. SIAM Journal on Applied Mathematics, 35(1), 68-82. for Loop Matchings. SIAM Journal on Applied Mathematics, 35(1), 68-82.
Pasmant, E. et al. (2011). ANRIL, a long, noncoding RNA, is an unexpected major Pasmant, E. et al. (2011). ANRIL, a long, noncoding RNA, is an unexpected major
hotspot in GWAS. FASEB journal : official publication of the Federation of hotspot in GWAS. FASEB journal : official publication of
American Societies for Experimental Biology, 25(2), 444-8.

American Societies for Experimental Biology, 25(2), 444-8.
Sankoff, D. (1985). Simultaneous Solution of the RNA Folding, Alignment and Protosequence Problems. SIAM J. Appl. Math., 45(5), 810-825.

Sato, K. et al. (2011). IPknot: fast and accurate prediction of RNA secondary structures with pseudoknots using integer programming. Bioinformatics, 27(13), i85-i93.

Sato, K. et al. (2012). DAFS: simultaneous aligning and folding of RNA sequences via dual decomposition. Bioinformatics, 28(24), 3218-3224.

Siegfried, N. A. et al. (2014). RNA motif discovery by SHAPE and mutational profiling (SHAPE-MaP). Nature Methods, 11(9), 959-965.

Spitale, R. C. et al. (2015). Structural imprints in vivo decode RNA regulatory mechanisms. Nature, 519(7544), 486-490.

mechanisms. Nature, 519(7544), $486-40$. Tan, Z. et al. (2017). TurboFold II: RNA structural alignment and secondary structure
prediction informed by multiple homologs. Nucleic Acids Research, $\mathbf{4 5}(20)$, prediction in $11570-11581$.

Torarinsson, E. et al. (2007). Multiple structural alignment and clustering of RNA sequences. Bioinformatics, 23(8), 926-932.

Turner, D. H. and Mathews, D. H. (2010). NNDB: the nearest neighbor parameter database for predicting stability of nucleic acid secondary structure. Nucleic Acids Research, 38(suppl_1), D280-D282.

Wu, F. et al. (1991). tat regulates binding of the human immunodeficiency virus trans-activating region RNA loop-binding protein TRP-185. Genes \& development, $\mathbf{5}(11), 2128-40$ 